\title{
Electron tunneling times
}

\author{
N. G. Kelkar \\ Departamento de Fisica, Universidad de los Andes, \\ Cra 1E, 18A-10, Bogotá, Colombia
}

\begin{abstract}
Tunneling is one of the most bizarre phenomena in quantum mechanics. An attempt to understand it led to the next natural question of how long does a particle need to tunnel a barrier. The latter gave rise to several definitions such as the phase, dwell, Larmor and traversal times among others. A short review of the evolution of these time concepts, followed by an account of experiments involving field-induced tunnel ionization and electron tunneling in a solid state junction is presented here. Whereas the former experiments use sophisticated techniques involving femtosecond laser pulses and determine the tunneling time by mapping the angle of rotation of the field vector to time, like the hands of a watch, the latter provides a simpler method through the measurement of current-voltage characteristics of the junction.

PACS numbers: 03.65.Xp,03.65.Sq,73.40.Sx,33.20.Xx
\end{abstract}

Talk given at the $2^{\text {nd }}$ Jagiellonian Symposium on Fundamental and Applied Subatomic Physics, Kraków, June 4 - 9, 2017 


\section{INTRODUCTION}

The definition of time has always intrigued philosophers and physicists equally so. Whereas in the opinion of philosophers such as Immanuel Kant, space and time are the framework within which the mind is constrained to construct its experience of reality, a more pragmatic view was to consider time as something that we use a clock to measure. In physics time appears as a parameter, be it through Newton's second law, $\mathbf{F}=d \mathbf{p} / d t$, in classical physics or the Schrödinger equation, $i \hbar \partial \Psi / d t=H \Psi$, in quantum mechanics. We may then ponder if there is a way to measure time without referring to the parametric time. In other words, is there an expression which represents a time interval without directly depending on the parameter $t$ ? The answer to this question indeed leads us to the quantum time concepts developed in connection with collisions or scattering in three dimensions (3D) and tunneling in one dimension (1D). As we shall see in the next sections, both these times in 3D and 1D are "interaction times" of the subatomic particles involved. Their definitions [1] follow from similar conceptual considerations and find meaning in physical processes [2, 3].

\section{EVOLUTION OF QUANTUM TIME CONCEPTS}

We are a long way from 1928 when Gamow published his pioneering work [4] on the tunneling of alpha particles in radioactive nuclei. Though tunneling seems to be a well understood phenomenon with ramifications in many branches of physics, the amount of time spent by a particle in tunneling remains controversial. One of the earliest papers [5] on the topic studied the time evolution of a wave packet and concluded that there is no appreciable delay in the transmission of the packet through the barrier. Though the question of tunneling time as such did not attract much attention for another 25 years, it is interesting to note that the dwell time concept which was proposed by Smith [6] in 1960 appeared earlier in a different guise in a 1938 paper by Kapur and Peierls [7] on the study of cross sections with resonances. The dwell time, $\tau_{D}$ (sometimes called residence time), is a stationary concept and corresponds to the time spent by a particle in a given region of space with interaction. Smith derived the collision time in three dimensions (3D) and extended it to the multichannel case of elastic scattering with resonance formation. He constructed a lifetime matrix $\mathbf{Q}$ which was related to the scattering matrix $\mathbf{S}$ as, $\mathbf{Q}=-i \hbar \mathbf{S} d \mathbf{S}^{\dagger} / d E$, 
such that the diagonal element $Q_{i i}$ gave the average lifetime of a collision beginning in the $i^{\text {th }}$ channel. In the one channel, elastic scattering case, this expression reduces to the phase time delay $\left(\tilde{\tau}_{\phi}(E)=d \delta / d E\right.$, with $\delta$ being the scattering phase shift) derived by Wigner and Eisenbud [8] earlier. However, whereas the expression due to Smith which is derived from a time delayed radial wave packet is consistent with a lifetime matrix which is Hermitian, any Eisenbud-type lifetime matrix violates time reversal invariance [9]. The collision time of Smith reduces in one-dimension to the dwell time in tunneling [10] which is given as, $\tau_{D}(E)=\int_{x_{1}}^{x_{2}}|\Psi(x)|^{2} d x / j$, where, $|\Psi(x)|^{2}$ gives the probability density and $j$ the current density for a particle tunneling through a potential barrier with energy $E=(\hbar k)^{2} / 2 m$. It is then natural to expect a relation between the dwell time $\left(\tau_{D}\right)$ and the phase time $\left(\tau_{\phi}\right)$ which was indeed derived in [11] and given by,

$$
\tau_{\phi}(E)=\tau_{D}(E)-\hbar[\Im m R / k] d k / d E .
$$

It was shown in the $3 \mathrm{D}$ case to be [12], $\tilde{\tau}_{\phi}(E)=\tilde{\tau}_{D}(E)-\hbar \mu\left[t_{R} / \pi\right] d k / d E$. The last term in (11) arises due to the interference of the incident and the reflected waves in front of the barrier and makes the phase time singular near threshold. For large energies however, the phase and dwell times are the same. A relation between the phase time delay and number of resonances can be found in [13].

In the years to follow, more definitions of tunneling time arose in different contexts. For example, considering the spin precession of an electron in a weak magnetic field, Buettiker [10] defined the Larmor time which was related to the expectation value of the spin operator and reduced to the dwell time in the particular case of a rectangular barrier. The generalized Buettiker-Landauer time was derived later [14] and given by $\tau_{\mathrm{BL}}=-\hbar \partial \ln |T| / \partial V$, which appears to be similar in form to the Pollak-Miller time [15, 16], $\tau_{\mathrm{PM}}=\hbar \partial \ln |T| / \partial E$. The four tunneling times: the Larmor time, Buettiker Landauer time, Wigner's phase time and Pollak-Miller time, originally derived from very different physical assumptions were derived in a unified manner within a Feynman path integral approach [19] using Gell Mann Hartle decoherence functionals. The total wave function was expressed as a sum over all possible "paths" with each path contributing a phase containing the action for that path. At this point we refer the reader to the review articles [1, 16 -18] and continue in the rest of the article to find out which of the tunneling time definitions correspond to physically measured times. 


\section{EXPERIMENTAL EXTRACTION OF ELECTRON TUNNELING TIMES}

The time spent by subatomic particles in tunneling potential barriers is usually estimated to be extremely small and beyond the reach of experimental precision. Using the calculated values of free electron Fermi energies and measured values of the work function, Hartman [20] estimated the phase times for metal - insulator - metal sandwiches for several different materials to be of the order of $10^{-16} \mathrm{~s}$.

Though a direct measurement of such small times does not seem feasible, the advent of intense laser fields has made measurements on the tunneling of bound electrons from atoms possible [21 23]. In [21], for example, an (intensity averaged) upper limit of 12 attoseconds on the tunneling delay time in strong field ionization using helium atoms was placed. More recently, the authors in [23] find that the time delay in tunneling is zero for helium and argon atoms within the experimental uncertainty of a few $10 \mathrm{~s}$ of attoseconds $\left(10^{-18} \mathrm{~s}\right)$. In this strong field ionization process, the electron tunnels through the potential created by a superposition of the atomic Coulomb potential and the laser field. The free electron is further accelerated by the laser field and the tunneling time is determined by measuring the electron momentum which depends on the strength of the field. In [24] the authors perform a comparison of the extracted electron tunneling times with various theoretical definitions and conclude that only the Larmor time and the probability distribution of tunneling times constructed using a Feynman Path Integral formulation are compatible with experiment.

Apart from these recent experiments, worth mentioning is also an earlier attempt [25] using field emission microscopy (FEM). Measuring the transversal momentum spread of the electrons (with the help of a field emission microscope), emitted by an isolated center of the tip, the electron-tunneling time related with the field ionization of this center could be deduced. The precision of the experiment lied in the preparation of ultra sharp silicon tips (radius of curvature $10-20 \mathrm{~nm}$ ) coated with 50 - $100 \mathrm{~nm}$ thick $\mathrm{CaF}_{2}: \mathrm{Sm}^{2+}$ layers which could be used as the field-emission sources, for which the tunneling current would be due to the field ionization of single isolated bivalent samarium dopant ions.

Coming now to the tunneling of electrons through a solid state junction, in [3] a novel method to extract the dwell times of electrons in metal - insulator - metal sandwiches from current - voltage (I-V) characteristics was presented. Tunneling of electrons in solid state junctions was studied earlier in [26]. Ref. [3] reported the I-V characteristics in a 
$\mathrm{Al} / \mathrm{Al}_{2} \mathrm{O}_{3} / \mathrm{Al}$ junction for temperatures ranging from 3.5 to $300 \mathrm{~K}$. The experimental data was then used to fit the barrier height and width (for a rectangular barrier) using a standard semiclassical model for the I-V relation from [27]. The fits led to a constant value of barrier width $s \sim 20.8 \AA$ and a continuous increase in the barrier height $V_{0}(T)$ from $1.799 \mathrm{eV}$ at $300 \mathrm{~K}$ to $1.83 \mathrm{eV}$ at $3.5 \mathrm{~K}$. Temperature dependence of the energy gap, $E_{g}(T)^{\exp }$ was also determined and allowed the authors to determine the average phonon frequency $\omega$. An excellent fit to $E_{g}(T)^{e x p}$ was obtained with an average phonon frequency, $\omega=2.05 \times 10^{13}$ $\sec ^{-1}[3]$, in close agreement with the value $\omega=2.24 \times 10^{13} \mathrm{sec}^{-1}$, determined from the speed of sound measurements using picosecond ultrasonic technique in amorphous $\mathrm{Al}_{2} \mathrm{O}_{3}$. Having gained confidence about the precision of the measurements from the above agreement of the phonon frequencies, the barrier parameters were then used to extract the temperature dependent dwell times in tunneling. The average dwell time $\tau_{D}$ was found to depend very weakly on temperature. The value of $\tau_{D}$ was found to be $3.6 \times 10^{-16} \mathrm{sec}$ at mid-barrier energies. Extrapolating the values of the measured times in the field ionization experiments (see Fig. 3 of Ref. [24]) to widths comparable to the above junction, the order of magnitude of the times is similar. The importance of [3] lies in the fact that knowledge of the tunneling time is obtained in a much simpler experiment as compared to [24]. An extension of [3] including dissipative effects was done in 28$]$.

In passing, we note a recent experiment [29] based on the merger of two fields [30] which have rapidly grown over the past few decades: optical lattices (artificial crystals bound by light) and Bose Einstein Condensates (BEC). In [29] a direct measurement of the tunneling delay time through the barriers of an optical lattice was performed by studying the time evolution of a Rubidium-87 BEC after a sudden displacement of the lattice. The authors report delay times of the order of tens of $\mu s$.

\section{CONCLUDING REMARKS}

We started the discussion in this article by asking if time which appears as a parameter $t$ can also be expressed as a quantity which does not explicitly depend on the parameter, $t$. Indeed, we saw different quantum time concepts which are defined in terms of the energy, wave function, energy dependent phases and flux of the tunneling particles. Apart from the definitions themselves, there exist relations which connect the times to quantities such as 
the density of states. The energy derivative of the scattering phase shift which gives the phase time delay for example, is given via the Beth Uhlenbeck formula by [31],

$$
\sum_{l} n_{l}(E)-n_{l}^{0}(E)=\sum_{l} \frac{2 l+1}{\pi} \frac{d \delta_{l}}{d E}
$$

where, $n_{l}(E)$ and $n_{l}^{0}(E)$ are the densities of states with and without interaction respectively. This relation leads to interesting interpretations involving "time advancement" or negative time delay in a scattering process involving unstable states [32]. Similar relations can also be derived in one dimension with the phase time defined in terms of the phase of the transmission amplitude in tunneling [33].

Finally, we note that tunneling processes are inherently connected to the survival and decay of unstable states [34]. This fact relates the tunneling times as well as the collision times to the survival probabilities of the unstable states. The half-life of a radioactive nucleus which mostly exhibits the classical exponential decay law can be shown to be given by the dwell time in the tunneling of alpha particles through a Coulomb barrier [2]. The very same dwell time concept can however be used to extract the non-exponential behaviour of the decay law at large times [35] as predicted by quantum mechanics [36]. Investigation of the quantum time concepts has indeed led to the understanding of time in its different guises.

[1] E. H. Hauge and J. A. Støvneng, Rev. Mod. Phys. 61, 917-936 (1989).

[2] N. G. Kelkar, H. M. Castañeda and M. Nowakowski, Europhys. Lett. 85, 20006-p1 - p5 (2009).

[3] E. J. Patiño and N. G. Kelkar, Appl. Phys. Lett. 107, 253502-1 - 4 (2015).

[4] G. Gamow, Z. Phys. 51, 204-212 (1928).

[5] L. A. MacColl, Phys. Rev. 40, 621 (1932).

[6] F. T. Smith, Phys. Rev. 118, 349-356 (1960).

[7] P. L. Kapur and R. Peierls, Proc. R. Soc. A. 277-295 (1938).

[8] E. P Wigner, Phys. Rev. 98, 145-147 (1955); E. P. Wigner and L. Eisenbud, Phys. Rev. 72, 29 (1947); L. E. Eisenbud, Ph.D. Thesis, Princeton University, unpublished (1948).

[9] N. G. Kelkar and M. Nowakowski, Phys. Rev. A 78, 012709 (2008).

[10] M. Büttiker, Phys. Rev. B 27, 6178-6188 (1983).

[11] H. G. Winful, Phys. Rev. Lett. 91, 260401 (2003). 
[12] N. G. Kelkar, Phys. Rev. Lett. 99, 210403-1 - 4 (2007).

[13] Zafar Ahmed and Sudhir R Jain, J. Phys A 37, 867 (2004).

[14] C. R. Leavens and G. C. Aers, Solid State Comm. 63, 1107 (1987).

[15] E. Pollak and W. H. Miller, Phys. Rev. lett. 53, 115 (1984).

[16] Alexandra S. Landsman and Ursula Keller, Phys. Rep. 547, 1 (2015).

[17] J.G. Muga, R. Sala Mayato, I.L. Egusquiza (Eds.), Time in Quantum Mechanics, Vol. 1, second ed., Springer, Berlin, 2007; G. Muga, A. Ruschhaupt, Adolfo del Campo (Eds.), Time in Quantum Mechanics, Vol. 2, Springer, Berlin, 2009.

[18] C.A.A. de Carvalho, H.M. Nussenzveig, Phys. Rep. 364, 83 (2002).

[19] N. Yamada, Phys. Rev. Lett. 93, 170401 (2004).

[20] T. E. Hartman, J. Appl. Phys. 33, 3427-3433 (1962).

[21] P. Eckle et al., Science 322, 1525-1529 (2008).

[22] D. Shafir et al., Nature 485, 343-346 (2012); O. Pedatzur et al., Nature Physics 11, 815-819 (2015).

[23] A. N. Pfeiffer et al., Nature Phys. 8, 76 (2012); A. N. Pfeiffer et al., Chem. Phys. 414, 84 (2013).

[24] A. S. Landsman et al., Optica 1, 343 (2014).

[25] S. K. Sekatskii and V. S. Letokhov, Phys. Rev. B 64, 233311-1 - 4 (2001).

[26] D. Esteve et al., Phys. Scripta T29, 121 (1989); P. Gueret, E. Marclay and H. Meier, Solid State Comm. 68, 977 (1988).

[27] John G. Simmons, J. Appl. Phys. 34, 1793-1803 (1963).

[28] N. G. Kelkar, D. Lozano Gómez and E. J. Patiño, Annals of Phys. 382, 11 (2017).

[29] A. Fortun et al., Phys. Rev. Lett. 117, 010401 (2016).

[30] O. Morsch and M. Oberthaler, Rev. Mod. Phys. 78, 179 (2006).

[31] E. Beth and G. E. Uhlenbeck, Physics 4, 915 (1937); K. Huang, Statistical Mechanics (Wiley, New York, 1963); G. Iannaccone, Phys. Rev. B 51, R4727 (1995).

[32] E. Kolomeitsev and D.N. Voskresensky, J. Phys. G 40, 113101 (2013); N. G. Kelkar, J. Phys. G 29, L1 - L8 (2003).

[33] V. Gasparian and M. Pollak, Phys. Rev. B 47, 2038 (1993).

[34] L. Fonda, G. C. Ghirardi and A. Rimini, Rep. Prog. Phys. 41, 587 (1978); F. Giacosa, Phys. Rev. A 88, 052131 (2013). 
[35] N. G. Kelkar, M. Nowakowski and K. P. Khemchandani, Phys. Rev. C 70, 024601-1 -4 (2004).

[36] K. Urbanowski, Eur. Phys. J. D 71, 118 (2017); K. Urbanowski, Eur. Phys. J. D 54, 25 (2009); F. Giacosa, Found. Phys. 42, 1262 (2012). 